\title{
Temporal dynamics of depression, cognitive performance and sleep in older persons with depressive symptoms and cognitive impairments: a series of eight single-subject studies
}

\author{
Marii Zuidersma, ${ }^{1}$ Astrid Lugtenburg, ${ }^{2}$ Willeke van Zelst, ${ }^{1}$ Fransje E. Reesink, ${ }^{3}$ \\ Peter Paul De Deyn, ${ }^{3}$ Fijanne Strijkert, ${ }^{4}$ (1) Sytse U. Zuidema, ${ }^{5}$ (i) \\ and Richard C. Oude Voshaar ${ }^{1}$ (1) \\ ${ }^{1}$ Department of Psychiatry, University of Groningen, University Medical Center Groningen, Interdisciplinary Center Psychopathology and Emotion regulation, \\ Groningen, The Netherlands \\ ${ }^{2}$ GGZ Drenthe Mental Health Institute, Department Old Age Psychiatry, Assen, The Netherlands \\ ${ }^{3}$ Department of Neurology, Alzheimer Research Center, University of Groningen, University Medical Center Groningen, The Netherlands \\ ${ }^{4}$ University Center for Geriatric Medicine, University Medical Center Groningen, The Netherlands \\ ${ }^{5}$ Department of General Practice and Elderly Care Medicine, University of Groningen, University Medical Center Groningen, Groningen, The Netherlands
}

Objectives: To investigate the presence, nature and direction of the daily temporal association between depressive symptoms, cognitive performance and sleep in older individuals.

Design, setting, participants: Single-subject study design in eight older adults with cognitive impairments and depressive symptoms.

Measurements: For 63 consecutive days, depressive symptoms, working memory performance and night-time sleep duration were daily assessed with an electronic diary and actigraphy. The temporal associations of depressive symptoms, working memory and total sleep time were evaluated for each participant separately with time-series analysis (vector autoregressive modeling).

Results: For seven out of eight participants we found a temporal association between depressive symptoms and/or sleep and/or working memory performance. More depressive symptoms were preceded by longer sleep duration in one person $(\mathrm{r}=0.39 ; \mathrm{p}<.001)$, by longer or shorter sleep duration than usual in one other person $(\mathrm{B}=0.49 ; \mathrm{p}<.001)$, by worse working memory in one person $(\mathrm{B}=-0.45 ; \mathrm{p}=.007)$, and by better working memory performance in one other person $(B=0.35 ; \mathrm{p}=.009)$. Worse working memory performance was preceded by longer sleep duration $(\mathrm{r}=-.35 ; \mathrm{p}=.005)$ in one person, by shorter or longer sleep duration in three other persons $(\mathrm{B}=-0.76 ; \mathrm{p}=.005, \mathrm{~B}=-0.61 ; \mathrm{p}<.001 ; \mathrm{B}=-0.34 ; \mathrm{p}=.002)$, and by more depressive symptoms in one person $(\mathrm{B}=-0.25 ; \mathrm{p}=.009)$.

Conclusion: The presence, nature and direction of the temporal associations between depressive symptoms, cognitive performance and sleep differed between individuals. Knowledge of personal temporal associations may be valuable for the development of personalized intervention strategies in order to maintain their health, quality of life, functional outcomes and independence.

Key words: depressive symptoms, working memory, idiographic, n-of-1, actigraphy

Correspondence should be addressed to: Marij Zuidersma; University of Groningen, University Medical Center Groningen Department of psychiatry; PO Box 30001 (CC72); 9700 RB, Groningen, The Netherlands. Phone: + 31 50 3610107; E-mail: m.zuidersma@umcg.nl. Received 11 Sep 2020; revision requested 31 Oct 2020; revised version received 10 Dec 2020; accepted 14 Jan 2021. First published online 15 March 2021.

\section{Introduction}

Older persons with both cognitive impairments and depressive symptoms are a vulnerable group at increased risk of functional dependence (Xiang and An, 2015), nursing home admission (Xiang and An, 2015), and adverse health outcomes (Mourao et al., 2016). Reciprocal associations between depression and cognitive performance 
have consistently been shown over longer periods. That is, depression is associated with an increased risk of accelerated cognitive decline (Shin et al., 2020; Byers and Yaffe, 2011), and cognitive impairments are associated with a worse course of depression (Van den Kommer et al., 2013). While most literature focused on long-term associations between depression and cognitive performance, depression and cognitive performance may also be associated in both directions from one day to the next. Getting more insight into the daily temporal associations between depression and cognitive performance may be valuable for the development of daily intervention strategies in order to maintain health, quality of life, functional outcomes and independence.

\section{Day-to-day associations between depression and cognitive performance}

Depressive symptoms (Rosmalen et al., 2012) and cognitive performance (Nesselroade and Salthouse, 2004; Allaire and Marsiske, 2005) both show high day-to-day variability, and thus may be temporally associated with each other on a daily basis. Depression may induce next-day deficits in cognitive performance, for example through worse nutrition and poor physical activity during the day (Meeusen, 2014), more alcohol consumption, or physiological alterations (e.g. decreased brain-derived neurotrophic factor-levels and inflammation [Kim et al., 2016]), while reduced cognitive performance may yield an emotional reaction resulting in next-day depressive symptoms.

\section{Sleep}

Sleep problems are common in late-life depression as well as cognitive impairments (Bao et al., 2017; Hu et al., 2017; Siddarth et al., 2020), and sleep quality and quantity show high day-to-day variability (Knutson et al., 2007; Dillon et al., 2015). It is therefore likely that sleep plays a role in the daily fluctuations of depression as well as cognitive performance.

It is well recognized that sleep plays a role in memory consolidation (Stickgold, 2005; Diekelmann, 2014), and sleep deprivation has been found to be associated with reduced neurocognitive performance and alterations in brain activation (reviewed by Durmer and Dinges, 2005), and reduced cerebral blood flow (Zhou et al., 2019). On the other hand, longer sleep duration has been associated with decreased physical activity levels (Stranges et al., 2008) and increased sleepiness (Horne, 1991), which may in turn result in worse next-day cognitive performance. According to our knowledge, only two observational studies in real life specifically examined the impact of sleep on next-day cognitive performance. The first study found that both more and less than average sleep was associated with reduced next-day cognitive performance (Gamaldo et al., 2010). The other study found that poor subjective (but not objective) sleep was associated with worse next-day cognitive performance in chronic pain patients, but better next-day cognitive performance in healthy persons (Curtis et al., 2018). The authors explained this latter finding by a state of hyperarousal due to sleep loss.

Depression may impact subsequent sleep problems through increased ruminative thinking (Takano and Tanno, 2011) and the associated autonomic arousal and emotional distress that associates with it (Harvey, 2002). A lack of sleep may result in next-day depressive symptoms by increased stress reactivity (Meerlo et al., 2008), or an increase in amygdala activity (Yoo et al., 2007). Supportive of this, a bi-directional association between sleep and affect was found with a systematic review of daily diary studies: worse sleep predicted worse affect and vice versa (Konjarski et al., 2018). Longer sleep duration, on the other hand, may also result in next-day depressive symptoms, for example through increased inflammation (Dowd et al., 2011), or decreased physical activity (Stranges et al., 2008).

\section{The single-subject approach in order to take into account individual differences}

Most previous studies on daily associations between depression, sleep and cognitive performance used multilevel analyses to evaluate daily associations within persons. As such, these studies still focused on group-averages and thus between-person associations. Such group-aggregated results can only be generalized to individual persons, under very strict conditions. That is, the average, the variance, the covariance and lagged covariance between variables should be exactly the same for all individuals, and no changes over time in these statistical characteristics should be present (assumption of ergodicity: Nesselroade, 1991; Hamaker et al., 2005; Molenaar and Campbell, 2009). It is very likely that the condition of ergodicity is not met for sleep, depression and cognitive performance. Consequently, findings from studies focusing on group-averages on these associations will not be applicable to individual persons. Single-subject studies analyze each individual separately, and are thus able to explore the temporal association of variables over time within an individual. Consequently, single-subject studies yield results that apply to the individual, which is essential in the presence of large inter-individual differences. Furthermore, they can account for the 
time-varying nature of sleep, depression and cognitive performance, by virtue of the multitude of repeated assessments.

\section{Objective of the present study}

The objective of the present study was to explore the temporal dynamic association of depressive symptoms, cognitive performance and sleep using a single-subject design in older persons with both depression and cognitive impairments. We hypothesized that the presence, nature (i.e. is it a positive or a negative association?), strength and direction (i.e. does $\mathrm{X}$ precede $\mathrm{Y}$ or does $\mathrm{Y}$ precede $\mathrm{X}$ ?) of the associations differ between persons.

\section{Methods}

\section{Design and participants}

This study, the "idiographic study on cognition, affect and sleep in the elderly" (i-CASE) comprised an idiographic single-subject study design in older persons with depressive symptoms and cognitive impairments. Each participant completed 63 assessments of depression, sleep, and cognitive functioning using a diary and actigraphy. Following the idiographic single-subject design, time-series of each participant were analyzed separately.

Between January 2016 and January 2019, participants were recruited from the University Center Psychiatry and the Memory Clinic of the University Medical Center Groningen, and a mental health care institute in Groningen (Lentis). Patients were eligible if they 1) were aged $\geq 60$ years, 2) had depressive symptoms defined as a major depressive disorder according to Diagnostic Statistical Manual (DSM) criteria or a 15-item Geriatric Depression Scale (GDS) score $\geq 4$, 3) had cognitive impairment defined as a diagnosis of dementia or mild cognitive impairment (MCI) according to the multidisciplinary team of the Memory Clinic, a Mini Mental State Examination (MMSE) score $<25$, or a Montreal Cognitive Assessment (MOCA) score < 26. Patients were excluded if they had a somatic disorder influencing short-term survival, had moderate to severe dementia according to a clinical dementia rating scale $\geq 2$, had a bipolar, psychotic or substance use disorder during the past 2 years, were not able to participate due to for instance functional or language impairments, or if the treating specialist deemed the patient mentally incompetent to give consent for study participation.

The study protocol was approved by the institutional review board at the University Medical Center Groningen, and written informed consent was obtained from each study participant (METc 2013/019)

\section{Procedures}

At baseline, a trained research assistant administered several questionnaires and interviews, which are described in the Supplemental file. After this, the study period of 63 consecutive days started. During the 63-day study period, participants filled out an electronic diary on a study laptop every evening within 1 hour before going to bed. The diary included self-report questionnaires and a computerized cognitive task battery. Participants were instructed to fill-out the questions and perform the cognitive task in a quiet place with minimal distraction. Additionally, participants wore a MotionWatch8 (CamnTech) during the whole study period of 63 days in order to estimate sleep.

\section{Variables in the time series analyses}

Depressive symptoms, cognitive performance and sleep were the endogenous variables that were assessed on a daily basis and used in the time series analysis. Endogenous variables are variables that are both determinant and outcome at the same time in the time series analysis.

For depressive symptoms the 8-item Patient Health Questionnaire (PHQ-8, Kroenke et al., 2009) was used. The PHQ-8 evaluates current presence and severity of eight out of the nine DSM-IV depressive symptoms (without suicidal ideation). For the present study, the item on sleep was also not included in order to reduce overlap with our measure for sleep. Each of the seven remaining items was rated on a 7-point Likert scale from (1 not present to 7 most severe). An average score was calculated by dividing the total score by the number of items, resulting in a possible score from 1 (not present) to 7 (most severe). For one participant (Participant 8), the PHQ-score was log-transformed to make it normally distributed.

Sleep was defined as the total time during the night-time sleep-period that was actually spent in sleep (total night-time sleep time [TST]) as derived from the MotionWatch8. TST was calculated by adding up each 30 -second epoch during the nighttime sleep-period that was defined as sleep.

Because working memory was previously found to co-vary with mood within persons (Riediger et al., 2011; Brose et al., 2012; Brose et al., 2014), we focused on working memory for the present analysis. Working memory was assessed every evening with the One Back Task (ONB) as part of the Cogstate Brief Battery (Hammers et al., 2012). For the present analyses, the reaction time for correct responses was the primary outcome of the ONB. These reaction times were transformed using a base 10 logarithmic transformation 
in order to make them normally distributed, and the inverse was taken so higher scores represent better scores.

\section{Data preparation}

A "time" variable was created, indicating the unit of the time series analysis (or assessment day, thus ranging from 1 till the number of the last day). Each unit in the time series consisted of sleep during the night and depression and cognitive performance during the following evening (see Figure 1). Missing values were imputed for each participant separately with the R-package Amelia. The number of missing values that were imputed for each person can be found in Supplemental Table 1. For some participants the length of the time series deviated from 64, because it was logistically more achievable to extend the study period or to stop earlier. We standardized the three endogenous variables for each person separately (so one unit in the variable was equivalent to one person-specific SD).

\section{Power}

For time-series analysis, power is derived from the number of repeated measures instead of the number of participants, as the analyses are done for each individual separately. It is difficult to make power analyses for time series analyses, because effect sizes as commonly used in conventional group-based study designs do not apply for the analyses of effects over time in single persons. This, because the direction of causality and the number of lagged influences in the system under investigation is usually unknown and bidirectional and feedback effects can be present as well (Brandt and Williams, 2007). Furthermore, the regression coefficients of a VAR cannot be interpreted individually, as they are part of a system of variables that are dynamically related to each other. As a result, "effect sizes" as commonly used in conventional study designs do not apply. Simulation studies have shown that time-series analysis can already be done with 30 repeated measures, although more observations yield more power and more reliable results (Lütkepohl, 2005). Often, a minimum number of 50 is used (Box et al., 1994). Although there is some progress about power calculations for $\mathrm{n}$-of-1 experimental designs (Percha et al., 2019), simulation studies must still be done to acquire more information about power analysis for $\mathrm{n}$-of-1 observational studies using VAR in the current field.

\section{Statistics}

The temporal dynamic associations between sleep, depression and cognitive performance were evaluated using vector autoregressive modeling (VAR) analyses. VAR analysis separates time-lagged effects from contemporaneous effects. Therefore, the model allows to make inferences about the temporal order of effects. Bi-directional and feedback effects can also be investigated because variables can be treated as determinant and outcome at the same time (i.e. endogenous variables). In the present study, the VAR model consisted of a system of three endogenous variables representing our three primary outcomes for sleep, depression and cognitive performance. Each of the endogenous variables was regressed on its own lagged values and the lagged values of the other endogenous variables, as well as on the contemporaneous and lagged exogenous variables.

We chose to include one lag in the model, which is equivalent to one day. This, because we expected the values of sleep, depression and cognitive performance of the previous day to be most relevant for their current values for most persons. This resulted in the following equations:

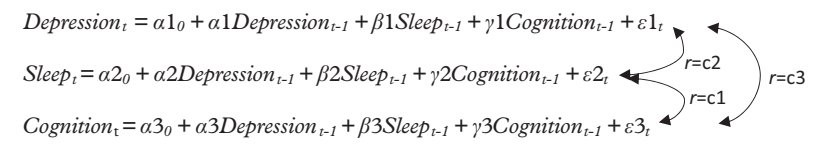

The parameters $\alpha 1, \beta 1$, and $\gamma 1$ were estimated for depression. For sleep the parameters $\alpha 2, \beta 2$ and $\gamma 2$ were estimated, and for cognition the parameters $\alpha 3, \beta 3$ and $\gamma 3$ were estimated. The residuals $\left(\varepsilon 1_{t}, \varepsilon 2_{t}\right.$ and $\left.\varepsilon 3_{t}\right)$ should be serially uncorrelated but can be contemporaneously correlated. The correlation between the residuals can be interpreted as the contemporaneous effects between the endogenous variables and are denoted c1, c2 and $\mathrm{c} 3$ in the equations (Brandt and Williams, 2007). The abovementioned equations result in the following model that was estimated for each participant (Figure 2):

In the present study, the r-package AutoVAR (Emerencia et al., 2016; van der Krieke et al., 2015) was used to perform the analyses. See Supplemental file for a description of AutoVAR.

Because some studies suggest that both more and less sleep may have negative effects (Gamaldo et al., 2010), we repeated the analyses using a variable for TST that was transformed in such a way that 1 unit increase represented a deviation (up or down) of 1 standard deviation (SD) from the person-specific average TST. In these analyses, the transformed TST variable was included as exogenous variable only (i.e. as an independent, but not as a dependent variable). This, because this variable was highly skewed and could not be normalized through transformation. Two exogenous variables for 


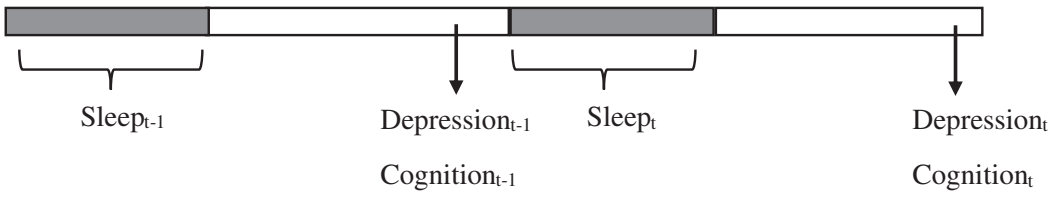

Figure 1. The timing of assessment of sleep, depression and cognition illustrated for 2 days within the 63-day study period ${ }^{1}$.

${ }^{1}$ Because the study period started with the evening assessment, 'time' $=1$ consisted only of data for depression and cognitive performance, but not for sleep. The first sleep assessment was therefore represented at 'time' $=2$. 'Time' $=64$ therefore represented the last sleep assessment, and no data for depression and cognitive performance. As a result of this data organization, a contemporaneous association of sleep with depression and/or cognitive performance would indicate an effect of sleep last night on depression and/or cognitive performance the subsequent evening. A lagged effect of sleep on depression and cognitive performance would indicate an effect of sleep the night before last night on depression and cognitive performance in the evening. The missing value for sleep at 'time $=1$ ', for depression and cognitive performance at 'time' $=64$, and any other missing values were imputed for each participant separately with the R-package Amelia.

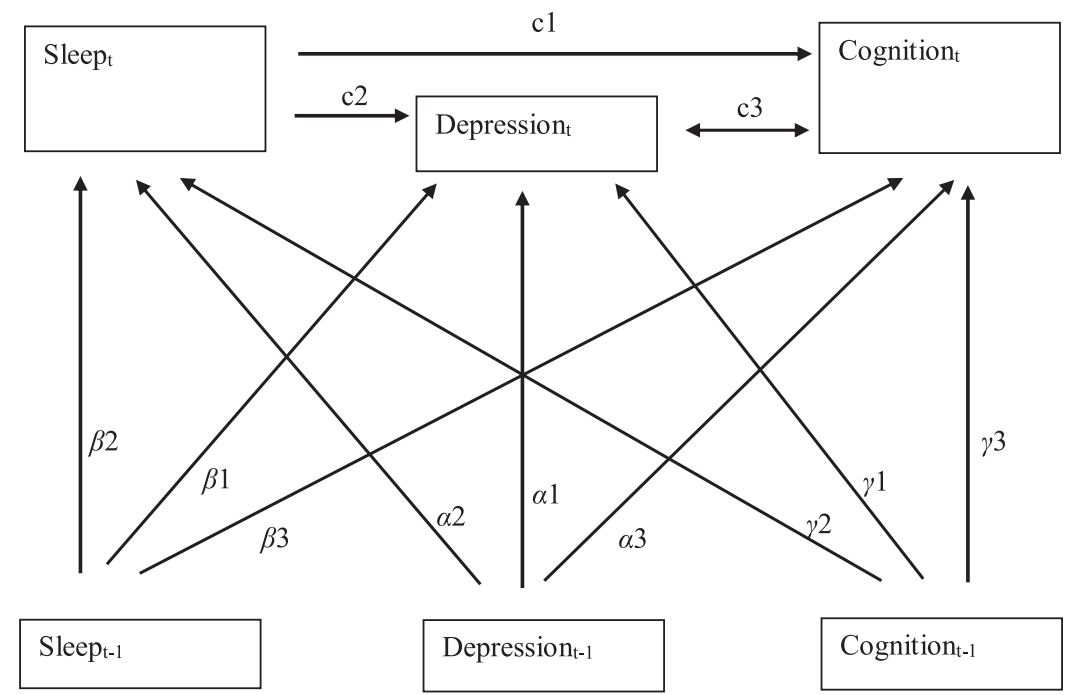

Figure 2. Estimated model for each participant ${ }^{1}$.

${ }^{1}$ The 3 arrows denoted with $\mathrm{c} 1,_{1} \mathrm{c} 2$ and $\mathrm{c}_{3}$ are the contemporaneous associations. Note that for the contemporaneous association of Depression $_{t}$ with Cognition $_{t}$ the direction of the association is unclear, because they are assessed right after each other. This association is therefore represented with a double-headed arrow. However, note that for the contemporaneous of Sleep $\mathrm{t}_{\mathrm{t}}$ with Depression $_{\mathrm{t}}$ and for Sleep $_{\mathrm{t}}$ with Cognition $\mathrm{n}_{\mathrm{t}}$ there may be a direction, because this is the association of sleep last night with depression and cognition in the evening. Therefore this association is represented with a one-headed arrow.

The vertical arrows denote the autoregressive effects $\alpha 1, \beta 2$ and $\gamma 3$.

The diagonal arrows denote the cross-lagged effects $\beta 1, \gamma 1, \alpha 2, \gamma 2, \alpha 3$, and $\beta 3$.

transformed TST were included in these models: at time $=\mathrm{t}$ and at time $=\mathrm{t}-1$.

The coefficients of the VAR equations cannot be interpreted individually because they are part of a dynamic system in which all variables are interrelated. That is, the net effect of a predictor may be different from its effect on a particular lag due to autoregressive effects and feedback loops. Therefore, we additionally used the techniques of Granger Causality and Cumulative Orthogonalized Impulse Response function (COIRF) analysis (Granger, 1969; Lütkepohl, 2005; Brandt and Williams, 2007). The Granger Causality technique evaluates whether current values of $\mathrm{Y}$ are (significantly) predicted by previous values of $\mathrm{X}$ beyond previous values of $\mathrm{Y}$ only. We performed COIRF to evaluate the dynamic effect size of those associations between endogenous variables that showed a significant Granger Causality or contemporaneous association. COIRF evaluates the net strength of the associations, by visualizing the cumulative effect of a shock (or 1 SD increase) in one variable on the other variables over a certain period of time (in our case 10 days). It incorporates potential effects of different lags, autoregressive effects and feedback loops, and takes into account both contemporaneous and lagged associations. It assumes a specific ordering of the contemporaneous association. For the associations of sleep with depression and cognition, the ordering of the contemporaneous 
association was always from sleep to depression/ cognition because this was inherent to the design (see Figure 1). Therefore, we examined only this order for the association of sleep with depression and cognition. For the association between depression and cognition the order of the contemporaneous association was unknown (because they were assessed right after one other in the evening diary, see Figure 1), so we examined both orders. COIRF analyses were performed using the irf function of the vars package in $\mathrm{R}$.

In order to estimate whether the presence and nature of the associations differed between persons, we compared the $r$-coefficients of the contemporaneous associations, and the VAR estimates between persons using Fisher Z's formula: $Z=\left(\mathrm{z}_{1}-\mathrm{z}_{2}\right) /$ (square root of $\left[\left(1 / \mathrm{N}_{1}-3\right)+\left(1 / \mathrm{N}_{2}-3\right)\right]$, where $\mathrm{z}=.5[\ln (1+\mathrm{r})-\ln (1-\mathrm{r})]$, and $\mathrm{N}$ is the length of the time series (t) (Fisher, 1925). All pairs of associations with $\mathrm{z}<-2.58$ or $\mathrm{z}>2.58$ were deemed statistically significant (which is analogous to $\mathrm{p}<.01$ ).

Because multiple tests were done, we used a more conservative alpha of .01 for indicating statistical significance.

\section{Results}

\section{Sample}

Twelve older persons participated in the i-CASE study. Two participants (participant 1 and 3) were excluded because their depressive symptoms were already in remission at the start of the study, one participant (Participant 6) refused to do the cognitive test battery, and for one participant (Participant 12) the multidisciplinary team of the Memory Clinic could not confirm MCI. Therefore, eight participants were included in the present analysis: four women and four men, aged 61-83 years, with varying levels of education, Geriatric Depression Scale scores ranged between 5 and 12, and Pittsburgh Sleep Quality Index scores ranged between 5 and 18. During the whole study period, participants slept on average 6.1 to 8.9 hours per night, scored between 1.3 and 5.0 on a scale from 1 to 7 on depressive symptoms, and it took them on average 618 to $912 \mathrm{~ms}$ for a correct response on the one-back task (see Supplemental Table 1).

\section{Results of the VAR analyses: Granger causality and contemporaneous associations}

The results of the VAR analyses accompanied with the Granger causality tests demonstrated differences in presence, nature (i.e. is it a positive or negative association), and direction (i.e. does $\mathrm{X}$ precede $\mathrm{Y}$ or does $\mathrm{Y}$ precede $\mathrm{X}$ ?) of the associations between participants, which we describe below (see Figure $3 \mathrm{~A}$ to $3 \mathrm{~F}$ and $4 \mathrm{~A}$ to $4 \mathrm{D}$ for lagged associations, and Table 1 for contemporaneous associations).

EFFECTS ON DEPRESSIVE SYMPTOMS

For three out of eight participants, significant effects on depressive symptoms were found. More sleep last night preceded more depressive symptoms in the following evening in one participant (Participant $10 ; \mathrm{r}=.39 ; \mathrm{p}<.001$; see Table 1 , results of the contemporaneous associations). This effect in participant 10 was significantly different from that in participants 2 and 11 (Fisher Z: -4.04 and 2.70, see Supplement 2). For none of the participants sleep the night before last night significantly granger caused depressive symptoms in the evening (Figure 3A). Better working memory performance in the evening granger caused less depressive symptoms the following evening in Participant 7 (so indicating significant Granger Causality: $\mathrm{B}=-0.45 ; \mathrm{p}($ Granger causality $)=.007)$. In contrast, better working memory performance in the evening granger caused more depressive symptoms the following evening in Participant $4(B=0.35$; $\mathrm{p}($ Granger causality $)=.009$; Figure $3 \mathrm{~B})$. Fisher Z test supported the difference in presence and nature of this association in participants 4 and 7: the association in these participants differed significantly from each other, but also from most other participants (see Supplement 2).

EFFECTS ON TOTAL Night-TIME SLEEP DURATION None of the participants had significant granger causality on total night-time sleep duration (see Figure 3C and D).

EFFECTS ON WORKING MEMORY PERFORMANCE Two out of eight participants had significant effects on working memory performance. For Participant 8, more hours of sleep last night $(\mathrm{r}=-0.35 ; \mathrm{p}=.005$; see Table 1) as well as the night before last night $(\mathrm{B}=-0.30 ; \quad \mathrm{p}$ (Granger $\quad$ causality $)=.008 ;$ see Figure $3 \mathrm{E}$ ) preceded worse working memory performance. This effect in participant 8 was significantly different from that in participants 4 and 11 (Fisher Z: 3.61 and -3.81, see Supplement 2). For Participant 11, more depressive symptoms yesterday evening granger caused worse working memory performance the following evening $(B=-0.25$; $\mathrm{p}($ Granger causality $)=.009$; Figure $3 \mathrm{~F})$. This effect in participant 11 was significantly different from that in participant 2 (Fisher Z: 4.30, see Supplement 2). 

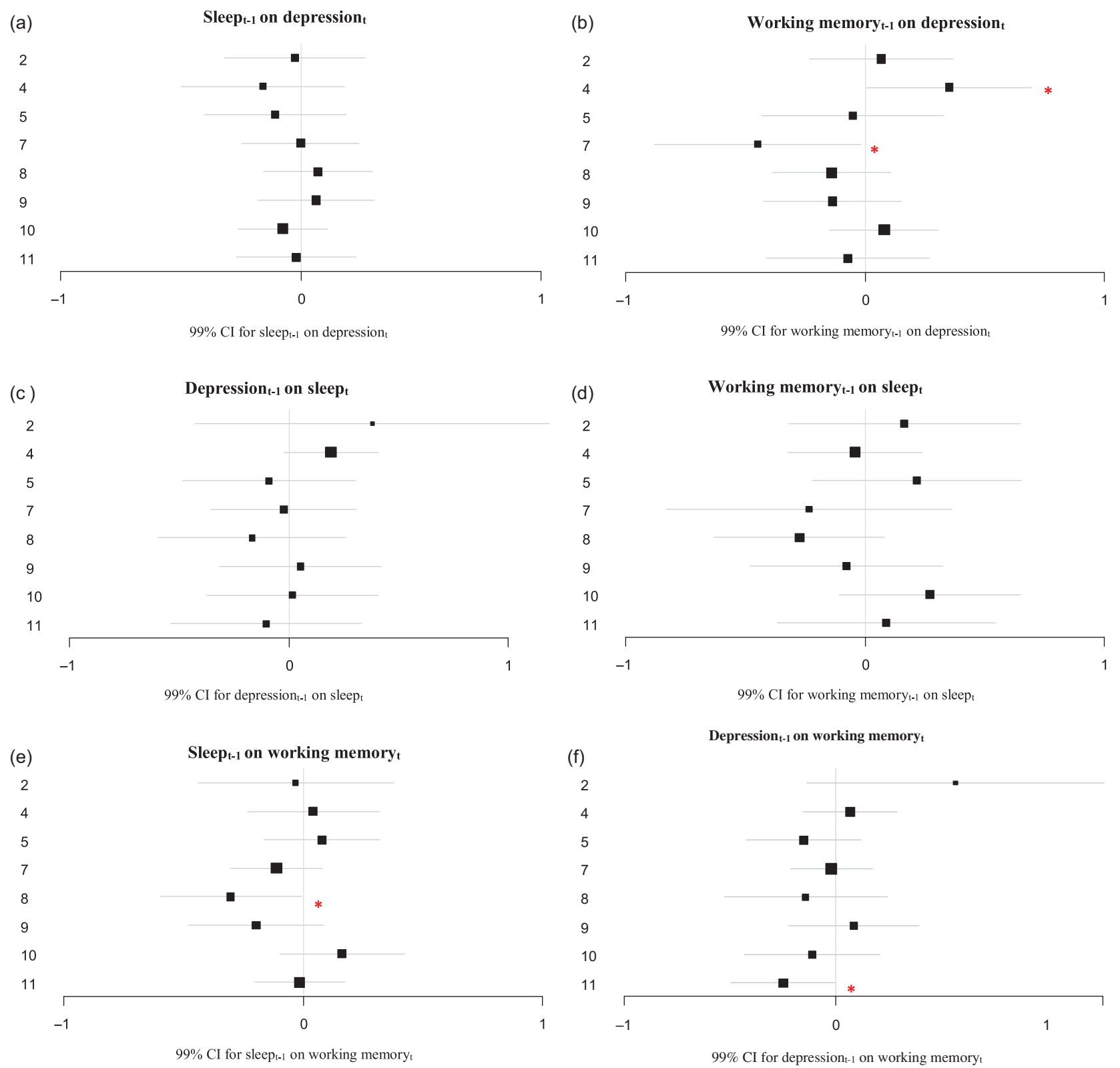

Figure 3. (A to F) Estimates and $99 \% \mathrm{Cl}$ of the lagged effects between sleep, depression and working memory resulting from the VAR analyses. The asterisks indicate a significant Granger causality effect $(p<.01)$. For Participant 8 the depression score was logtransformed to achieve normality. Missing values were imputed with Amelia.

EFFECTS OF MORE OR LESS THAN AVERAGE SLEEP The transformed sleep variable could not be entered as an endogenous variable in the VAR models, because it was highly skewed and could not be normalized through transformation. Therefore, two variables representing more or less than average sleep at $\mathrm{t}$ and at $\mathrm{t}-1$ respectively were entered as exogenous variables in the VAR model. For two participants more or less than average sleep preceded depressive symptoms. For Participant 2, more or less hours of sleep than average preceded more depressive symptoms the following evening $(\mathrm{B}=0.49 ; \mathrm{p}<.001$, Figure $4 \mathrm{~A})$. This effect in participant 2 was significantly different from that in most other participants (see Supplement 2). For Participant 4, more or less hours of sleep than average preceded less depressive symptoms the evening of the day thereafter (so, with an extra day in-between; $B=-0.99 ; p<.001$; Figure $4 B$ ). For three participants more or less than average sleep preceded worse working memory performance (Participants 2, 9 and 11; $\mathrm{B}=-0.76 ; \mathrm{p}=.005$, $\mathrm{B}=-0.61 ; \mathrm{p}<.001$ and $\mathrm{B}=-0.34 ; \mathrm{p}=.002$ respectively; Figure 4C). This effect in participants 2 and 9 indeed were significantly different from that in the 4 participants without an effect, but in 
Table 1. Contemporaneous associations resulting from the VAR analyses ${ }^{1,2}$

\begin{tabular}{|c|c|c|c|}
\hline \multirow[b]{2}{*}{ PARTICIPANT } & \multicolumn{3}{|c|}{ CORRELATION COEFFICIENT (P-VALUE) } \\
\hline & $\begin{array}{l}\text { SLEEP }_{T} \text { WITH } \\
\text { DEPRESSION }_{T}\end{array}$ & $\begin{array}{l}\text { SLEEP }_{\mathrm{T}} \text { WITH WORKING } \\
\text { MEMORY }_{\mathrm{T}}\end{array}$ & $\begin{array}{c}\text { DEPRESSION }_{\mathrm{T}} \text { WITH WORKING } \text { MEMORY }_{\mathrm{T}}\end{array}$ \\
\hline 2 & $-.40(.015)$ & $.10(.571)$ & $-.20(.231)$ \\
\hline 4 & $.02(.846)$ & $.23(.026)$ & $-.13(.212)$ \\
\hline 5 & $.28(.025)$ & $-.10(.446)$ & $-.07(.608)$ \\
\hline 7 & $.08(.547)$ & $-.02(.859)$ & $.01(.944)$ \\
\hline 8 & $.03(.845)$ & $-.35(.005)^{*}$ & $-.05(.672)$ \\
\hline 9 & $.05(.721)$ & $-.14(.273)$ & $-.12(.355)$ \\
\hline 10 & $.39(<.001)^{* *}$ & $-.05(.672)$ & $-.15(.207)$ \\
\hline 11 & $-.04(.747)$ & $.30(.013)$ & $-.12(.332)$ \\
\hline
\end{tabular}

Note. VAR = vector autoregressive modeling.

${ }^{1}$ The correlation coefficients indicate the correlation between the residuals of the VAR models.

${ }^{2}$ Missing values were imputed by the $r$ package Amelia

*p $<.01 ; * * \mathrm{p}<.001$

(a)

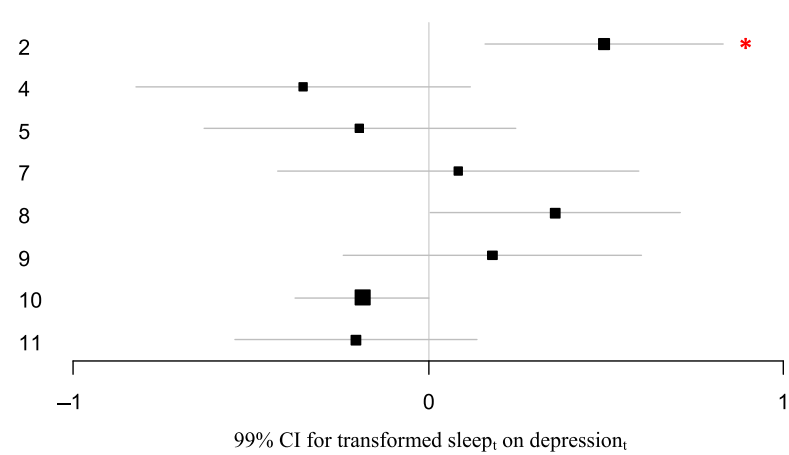

(c) Transformed sleep on working memory $_{t}$

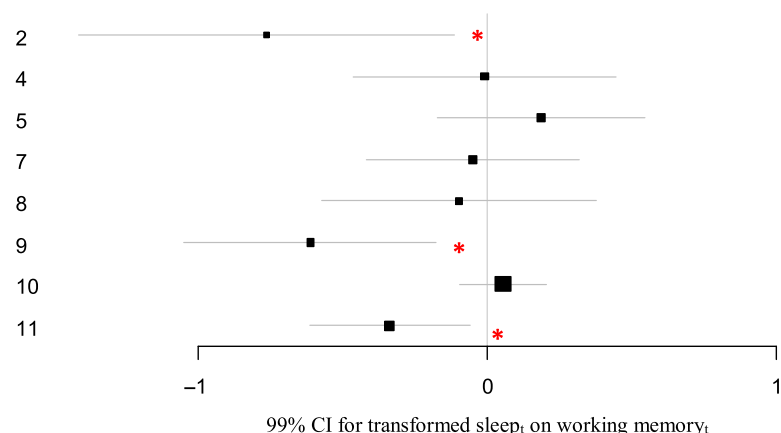

(b)

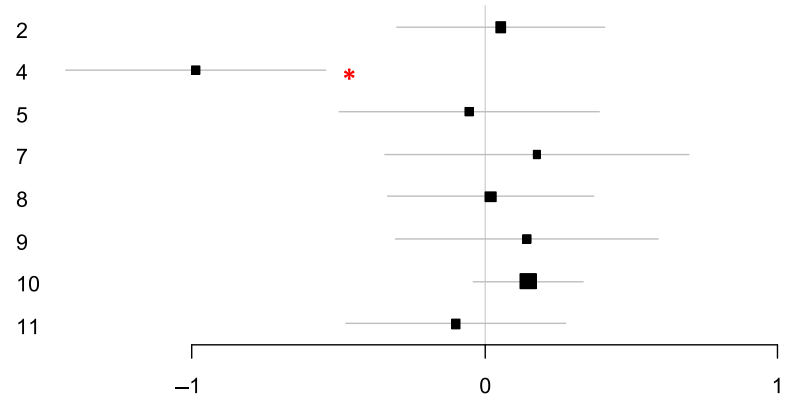

$99 \%$ CI for transformed sleep $\mathrm{t}_{\mathrm{t}-1}$ on depression $\mathrm{t}_{\mathrm{t}}$

(d) Transformed sleep $\mathrm{t}_{\mathrm{-}-1}$ on working memory

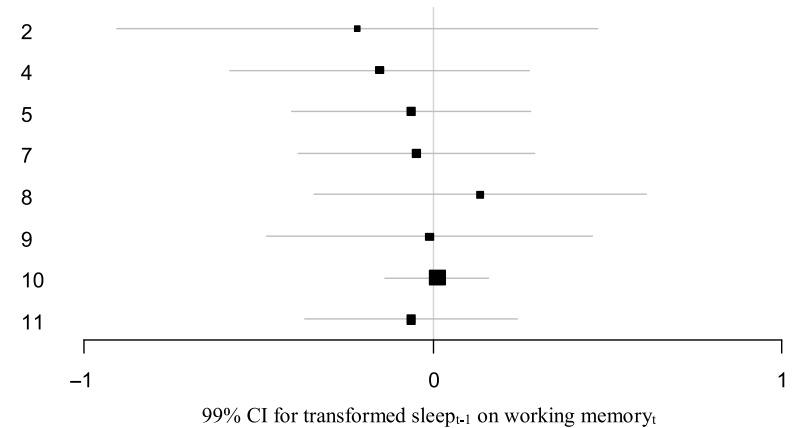

Figure 4. (A-D) Estimates and $99 \% \mathrm{Cl}$ of the lagged effects of more or less than average sleep on depression and working memory resulting from the VAR analyses.

The asterisks indicate a significant VAR effect $(p<.01)$. For Participant 8 the depression score was logtransformed to achieve normality. For participant 10 , the variables for depression and working memory were logtransformed to achieve normality. Missing values were imputed with Amelia.

participant 11 the effect differed significantly from that in participants 2 and 5 only (see Supplement 2). For none of the participants more or less than average sleep preceded worse working memory performance the evening of the day thereafter (so, with an extra day in between; see Figure 4D).

\section{Results of the cumulative orthogonalized impulse response function analysis (COIRF)}

The COIRF analyses showed differences in nature and direction of the associations between depression and working memory over a period of 10 days while incorporating potential effects of different 
lags, autoregressive effects and feedback loops (see Supplemental Table 2, and Supplemental Figure 1). To illustrate, for participant 4, a $1 \mathrm{SD}$ shock (increase) in working memory resulted in a 0.51 or 0.31 SD increase in depression over a 10-day period (depending on which order for the assumed contemporaneous association was chosen), while for participant 7 , it resulted in a 0.59 or $0.58 \mathrm{SD}$ decrease in depression (although the associations were not statistically significant, see Supplemental Table 2). So, the nature of the association differed between these two participants. Furthermore, in participant 11 the direction of the association differed from that of Participants 4 and 7 (i.e. a 1 SD shock in depression was associated with a 0.35 or 0.26 SD decrease in working memory over the next 10 days [so from depression to working memory vs. the other way around]). Furthermore, a shock in sleep did not significantly predict changes in working memory in participant 8 nor depression in participant 10 (see Supplemental Table 2, and Supplemental Figure 1). We were not able to evaluate the impact of a shock in the transformed sleep variable (representing the effect of a 1 SD increase or decrease in sleep) on depression or working memory because this sleep variable could not be normalized through transformation, and therefore not entered as an endogenous variable in the VAR model and thus also not in the COIRF model.

\section{Discussion}

\section{Summary of findings}

This series of single-subject studies in eight older persons with depressive symptoms and cognitive impairments found that the presence, nature, and direction of the daily temporal association between depressive symptoms, working memory and sleep differed between persons. This suggests that different persons may need different types of interventions in order to alleviate depressive symptoms.

\section{Interpretation of findings and clinical implications}

DEPRESSIVE SYMPTOMS AND COGNITIVE PERFORMANCE

For one of the eight participants more depressive symptoms on a given day preceded worse working memory performance the next day. For this participant, one SD increase in depressive symptoms on a given day was associated with about $0.3 \mathrm{SD}$ decrease in working memory over a period of 10 days (see Supplemental Table 2, participant 11). For persons showing such an association, effective depression treatment may perhaps enhance cognitive performance. For one other participant the direction of the association was reversed: worse working memory on a given day preceded more depressive symptoms the next day. Being aware of the cognitive deficits may have resulted in an increase in depressive symptoms for this person. Although the cumulative effect of decreased working memory on depressive symptoms over a 10-day period did not reach statistical significance for this person, a psychological intervention to increase the adjustment to the cognitive impairments may perhaps be helpful for persons who do show such an association (e.g. Regan and Varanelli, 2013).

AsSOCIATIONS OF SLEEP WITH DEPRESSIVE SYMPTOMS AND COGNITIVE PERFORMANCE In two out of eight participants changes in sleep preceded changes in depressive symptoms, but the nature of this relation differed between the two participants. Longer sleep duration preceded depressive symptoms the following day in one participant, although the cumulative effect of longer sleep duration on depressive symptoms over a period of 10 days was statistically not significant. In another participant shorter or longer sleep duration than average for this person preceded depressive symptoms. Unfortunately, we were not able to evaluate the cumulative effect of shorter or longer sleep duration on depressive symptoms over a period of 10 days, because this variable was highly skewed (and could not be entered as an endogenous variable). A previous nomothetic study also found longer or shorter sleep duration than average to be associated with worse depressive symptoms (Van Mill et al., 2014).

For none of the participants, changes in depression were associated with changes in sleep the following night, which is in contrast to a review that reported bi-directional effects between affect and sleep (Konjarski et al., 2018). This may be explained by the fact that some symptoms of depression may result in more sleep (e.g. loss of energy/fatigue), while other symptoms may result in less sleep (e.g. ruminative thinking).

Longer sleep duration was associated with worse next-day working memory performance in one out of eight participants. However, the cumulative effect of longer sleep duration on working memory over a 10-day period was not significant for this participant. In three other participants, shorter and longer sleep duration than average was associated with worse next-day working memory performance. This suggests that too short and too long sleep duration may both impact cognitive performance in the same person. 
For persons in whom longer sleep duration has adverse effects sleep restriction therapies may perhaps be beneficial (Lancee et al., 2019), while persons for whom longer or shorter sleep than usual has adverse effects may benefit from combined strategies to improve sleep as well as restrict sleep duration within a certain limit (e.g. Gebara et al., 2018; Gee et al., 2019; Lancee et al., 2019).

\section{Implications for clinical care}

This study showed that different persons may benefit from different interventions or advices. Implementing ambulatory diary and sensor assessments in clinical care settings may therefore help formulate patient-specific interventions or advices. However, it remains unclear to what extent it is feasible to equip a large number of patients with ambulatory sensors. Currently, technologies are increasingly being developed to enable this. Clinical care applications that implement single-subject analyses in clinical care settings to help in the diagnostic process are being developed (Fernandez et al., 2017; Fisher et al., 2019; Bos et al., 2019, conference abstract). In order to support this, tools to automatically preprocess and analyze data are being developed. Examples of this are the development of AutoVar to automatically perform VAR analysis (Emerencia et al., 2016; van der Krieke et al., 2015), the development of an rpackage that automatically preprocesses actiwatch data (ACTman: Kunkels et al., 2020), and the development of a platform that integrates data from sensors and diaries (Physiqual; Blaauw et al., 2016). Furthermore, implementing single-subject research in clinical care will also be more feasible when it is rewarding for patients to participate. Personalized feedback encourages shared decision making and patient empowerment, and appears particularly motivating to increase compliance in large samples (Kramer et al., 2014; Van der Krieke et al., 2017; Van Roekel et al., 2017). So, in the near future diary and sensor assessments may be implemented in clinical care settings.

\section{Methodological considerations}

The present study has several strengths. First, the intensive longitudinal assessment for each participant made it possible to evaluate temporal associations at the level of the individual patient. Second, although the sample consisted of older persons with cognitive impairments, adherence was relatively high, suggesting that this patient group is capable of performing daily assessments. This study has also some methodological challenges. First, as inherent to the single-subject study design, the results are not generalizable to other patients. However, generalizability was not an objective of the present study as the aim focused on finding patterns in individual persons. Second, like in nomothetic studies, the found associations in the present study are not necessarily causal, but may be explained by other unmeasured (fluctuating) variables. Third, the associations found by the present study do not say something about long-term effects, such as the course of depressive episodes. Instead of this, information about daily associations may be helpful for defining personalized daily intervention strategies, which may also have long-term effects (e.g. Van Roekel et al., 2017). Fourth, it remains unclear to what extent unreliability of each instrument may have influenced the current findings. Psychometric studies on single individuals should therefore be topic of future single-subject research. Fifth, we had to transform depression data for participant 8, while for the other participants untransformed data was used. This may have affected the coefficients for this participant, and limited comparison to the coefficients of the other participants. However, we chose not to transform data of all participants, because the assumptions were met with the untransformed data, so transforming is not advised (Feng et al., 2014).

\section{Conclusion}

The presence, nature and direction of the associations between sleep, depressive symptoms and cognitive performance differ between persons. Getting more insight into these daily temporal associations for a specific patient may guide the treating specialist in formulating an intervention strategy, which may help maintain the patients' health, quality of life, functional outcomes and independence.

\section{Conflict of interest}

None.

\section{Source of Funding}

This work was supported by Stichting MIND (project code 2013 6740) and Alzheimer Nederland (project code WE 09-2014-01).

\section{Description of authors' roles}

$\mathrm{MZ}, \mathrm{RCOV}$ and AL drafting first version of manuscript and substantially contributed to the design and conception of the work, all authors: critically revised the manuscript, read and approved the final version of the manuscript. 


\section{Acknowledgements}

We would like to thank all the participants of this study for their participation. We would like to thank Elisabeth $\mathrm{H}$ (Elske) Bos for her kind advice with the analyses.

\section{Supplementary material}

To view supplementary material for this article, please visit https://doi.org/10.1017/S104161 0221000065

\section{References}

Allaire, J. C. and Marsiske, M. (2005). Intraindividual variability may not always indicate vulnerability in elders' cognitive performance. Psychology and Aging, 20, 390-401. DOI: $10.1037 / 0882-7974.20 .3 .390$

Bao, Y. P. et al. (2017). Cooccurrence and bidirectional prediction of sleep disturbances and depression in older adults: meta-analysis and systematic review. Neuroscience E Biobehavioral Reviews, 75, 257-273. doi: 10.1016/j .neubiorev.2017.01.032.

Blaauw, F. J. et al. (2016). Let's get Physiqual-An intuitive and generic method to combine sensor technology with ecological momentary assessments. Fournal of Biomedical Informatics, 63, 141-149. doi: 10.1016/j.jbi.2016.08.001

Bos, F. M. et al. (2019). Developing a flexible interface to generate personalized diaries in mental health care. Support Health Technology, 9, 3.

Box, G. E.P., Jenkins, G. M. and Reinsel, G. C. (1994) Time series analysis: Forecasting and control. 3rd ed. Englewood Cliffs, NJ: Prentice Hall.

Brandt, P. T. and Williams, J. T. (2007). Multiple Time Series Models. Thousand Oaks, CA: Sage Publications. DOI: $10.4135 / 9781412985215 . n 1$

Brose, A., Lövdén, M. and Schmiedek, F. (2014). Daily fluctuations in positive affect positively co-vary with working memory performance. Emotion, 14, 1-6. DOI: 10.1037/ a0035210

Brose, A., Schmiedek, F., Lövdén, M. and Lindenberger, U. (2012). Daily variability in working memory is coupled with negative affect: the role of attention and motivation. Emotion, 12, 605-617. doi: 10.1037/a0024436.

Byers, A. L. and Yaffe, K. (2011). Depression and risk of developing dementia. Nature Reviews Neurology, 7, 323.

Curtis, A. F., Williams, J. M., McCoy, K. J. M. and McCrae, C. S. (2018). Chronic pain, sleep and cognition in older adults with insomnia: a daily multilevel analysis. Fournal of Clinical Sleep Medicine, 14, 1765-1772. doi: $10.5664 /$ jcsm.7392.

Diekelmann, S. (2014). Sleep for cognitive enhancement. Frontiers in Systems Neuroscience, 8, 46. doi: 10.3389/fnsys .2014 .00046

Dillon, H. R., Lichstein, K. L., Dautovich, N. D., Taylor, D. J., Riedel, B. W. and Bush, A. J. (2015). Variability in self-reported normal sleep across the adult age span. The fournals of Gerontology Series B: Psychological Sciences and Social Sciences, 70, 46-56. doi: 10.1093/ geronb/gbu035.

Dowd, J. B., Goldman, N. and Weinstein, M. (2011). Sleep duration, sleep quality, and biomarkers of inflammation in a Taiwanese population. Annals of Epidemiology, 21, 799-806. doi: 10.1016/j.annepidem.2011 .07 .004

Durmer, J. S. and Dinges, D. F. (2005). Neurocognitive consequences of sleep deprivation. Seminars in Neurology, 25, 117-129. DOI: $10.1055 / \mathrm{s}-2005-867080$

Emerencia, A. C., van der Krieke, L., Bos, E. H., de Jonge, P., Petkov, N. and Aiello, M. (2016). Automating Vector Autoregression on Electronic Patient Diary Data. IEEE Fournal of Biomedical and Health Informatics, 20, 631-643. doi: 10.1109/JBHI.2015.2402280

Feng, C. et al. (2014). Log-transformation and its implications for data analysis. Shanghai Archives of Psychiatry, 26, 105-109. doi: 10.3969/j.issn.1002-0829 2014.02.009.

Fernandez, K. C., Fisher, A. J. and Chi, C. (2017). Development and initial implementation of the dynamic assessment treatment algorithm (DATA). PLoS ONE, 12, e0178806. doi: 10.1371/journal.pone.0178806

Fisher, R. A. (1925). Statistical Methods for Research Workers. Edinburgh, Scotland: Oliver and Boyd.

Fisher, A. J. et al. (2019). Open trial of a personalized modular treatment for mood and anxiety. Behaviour Research and Therapy, 116, 69-79. doi: 10.1016/j.brat .2019 .01 .010

Gamaldo, A. A., Allaire, J. C. and Whitfield, K. E. (2010). Exploring the within-person coupling of sleep and cognition in older African Americans. Psychology and Aging, 25, 851-857. doi: 10.1037/a0021378

Gebara, M. A. et al. (2018). Effect of insomnia treatments on depression: a systematic review and metaanalysis. Depress Anxiety, 35, 717-731. doi: 10.1002/da .22776

Gee, B., Orchard, F., Clarke, E., Joy, A., Clarke, T. and Reynolds, S. (2019). The effect of non-pharmacological sleep interventions on depression symptoms: a metaanalysis of randomised controlled trials. Sleep Medicine Reviews, 43, 118-128. doi: 10.1016/j.smrv.2018.09.004

Granger, C. W. J. (1969). Investigating causal relations by econometric models and cross-spectral methods. Econometrica, 37, 424-438. DOI: 10.2307/1912791

Hamaker, E. L., Dolan, C. V. and Molenaar, P. C. M. (2005). Statistical modeling of the individual: Rationale and application of multivariate stationary time series analysis. Multivariate Behavioral Research, 40, 207-233. doi: 10.1207/ s15327906mbr4002_3

Hammers, D. et al. (2012). Validity of a brief computerized cognitive screening test in dementia. Fournal of Geriatric Psychiatry and Neurology, 25, 89-99. DOI: 10.1177/ 0891988712447894

Harvey, A. G. (2002). A cognitive model of insomnia. Behaviour Research and Therapy, 40, 869e93. DOI: 10.1016/ s0005-7967(01)00061-4

Horne, J. A. (1991). Dimensions to sleepiness. In: T. H. Monk (Eds.), Sleep, Sleepiness and Performance (pp. 169-196). Chichester, New York, Brisbane, Toronto, Singapore: John Wiley \& Sons. 
Hu, M. et al. (2017). Sleep disturbance in mild cognitive impairment: a systematic review of objective measures. Neurological Sciences, 38, 1363-1371. doi: 10.1007/ s10072-017-2975-9

Kim, H. K., Nunes, P. V., Oliveira, K. C., Young, L. T. and Lafer, B. (2016). Neuropathological relationship between major depression and dementia: a hypothetical model and review. Progress in Neuropsychopharmacology $\mathbb{E}$ Biological Psychiatry, 67, 51-57. doi: 10.1016/j.pnpbp .2016 .01 .008

Knutson, K. L., Rathouz, P. J., Yan, L. L., Liu, K. and Lauderdale, D. S. (2007). Intra-individual daily and yearly variability in actigraphically recorded sleep measures: the CARDIA study. Sleep, 30, 793-796. DOI: 10.1093/sleep/30 .6 .793

Konjarski, M., Murray, G., Lee, V. V. and Jackson, M. L. (2018). Reciprocal relationships between daily sleep and mood: a systematic review of naturalistic prospective studies. Sleep Medicine Reviews, 42, 47-58. doi: 10.1016/j .smrv.2018.05.005

Kramer, I. et al. (2014). A therapeutic application of the experience sampling method in the treatment of depression: a randomized controlled trial. World Psychiatry, 13: 68-77. doi: 10.1002/wps.20090

Kroenke, K., Strine, T. W., Spitzer, R. L., Williams, J. B., Berry, J. T. and Mokdad, A. H. (2009). The PHQ-8 as a measure of current depression in the general population. Fournal of Affective Disorders, 114, 163-173. DOI: $10.1016 /$ j.jad.2008.06.026

Kunkels, Y. K., Knapen, S. E., Zuidersma, M., Wichers, M., Riese, H. and Emerencia, A. C. (2020). ACTman: Automated preprocessing and analysis of actigraphy data. Fournal of Science and Medicine in Sport, 23, 481-486. doi: 10.1016/j.jsams.2019.11.009

Lancee, J., Maric, M. and Kamphuis, J. H. (2019). Sleep restriction therapy may be effective for people with insomnia and depressive complaints: evidence from a case series. Behavioural and Cognitive Psychotherapy, 11, 1-6. doi: $10.1017 /$ S1352465819000705

Lütkepohl, H. (2005). New Introduction to Multiple Time Series Analysis. Berlin, Heidelberg: Springer-Verlag. doi: 10.1007/978-3-540-27752-1

Meerlo, P., Sgoifo, A. and Suchecki, D. (2008). Restricted and disrupted sleep: effects on autonomic function, neuroendocrine stress systems and stress responsivity. Sleep Medicine Reviews, 12, 197-210. doi: 10.1016/j.smrv .2007.07.007

Meeusen, R. (2014). Exercise, nutrition and the brain. Sports Medicine, 44 Suppl 1, S47-56. doi: 10.1007/s40279-0140150-5.

Molenaar, P. C. M. and Campbell, C. G. (2009). The new person-specific paradigm in psychology. Current Directions in Psychological Science, 18, 112-117. Doi: 10.1111/j.14678721.2009.01619.x

Mourao, R. J., Mansur, G., Malloy-Diniz, L. F., Castro Costa, E. and Diniz, B. S. (2016). Depressive symptoms increase the risk of progression to dementia in subjects with mild cognitive impairment: systematic review and meta-analysis. International fournal of Geriatric Psychiatry, 31, 905-911. doi: 10.1002/gps.4406.

Nesselroade, J. R. (1991). Interindividual differences in intraindividual change. In: L. M. Collins and J. L. Horns
(Eds.), Best methods for the analysis of change: Recent advances, unanswered questions, future directions (pp. 92-105). Washington, DC: American Psychological Association.

Nesselroade, J. R. and Salthouse, T. A. (2004) Methodological and theoretical implications of intraindividual variability in perceptual-motor performance. The fournals of Gerontology. Series B, Psychological Sciences and Social Sciences, 59, P49-P55. doi: 10.1093/geronb/59.2.P49

Percha, B., Baskerville, E. B., Johnson, M., Dudley, J. T. and Zimmerman, N. (2019). Designing robust n-of-1 studies for precision medicine: simulation study and design recommendations. Fournal of Medical Internet Research, 21, e12641. doi: 10.2196/12641

Regan, B. and Varanelli, L. (2013). Adjustment, depression, and anxiety in mild cognitive impairment and early dementia: a systematic review of psychological intervention studies. Internationa Psychogeriatrics, 25, 1963-1984. doi: 10.1017/S104161021300152X

Riediger, M., Wrzus, C., Schmiedek, F., Wagner, G. G. and Lindenberger, U. (2011). Is seeking bad mood cognitively demanding? Contra-hedonic orientation and working-memory capacity in everyday life. Emotion, 11, 656-665. doi: 10.1037/a0022756

Rosmalen, J. G., Wenting, A. M., Roest, A. M., de Jonge, P. and Bos, E. H. (2012). Revealing causal heterogeneity using time series analysis of ambulatory assessments: application to the association between depression and physical activity after myocardial infarction. Psychosomatic Medicine, 74, 377-386. doi: 10.1097/PSY $.0 \mathrm{~b} 013 \mathrm{e} 3182545 \mathrm{~d} 47$

Shin, M. (2020). Depressive symptoms with cognitive dysfunction increase the risk of cognitive impairments: analysis of the Korean Longitudinal study of Aging (KLoSA), 2006-2008. International Psychogeriatrics, doi: $10.1017 / \mathrm{S} 1041610220003622$

Siddarth, P. et al. (2020). Sleep quality, neurocognitive performance, and memory self-appraisal in middle-aged and older adults with memory complaints. International Psychogeriatrics, doi: 10.1017/S1041610220003324

Stickgold, R. (2005). Sleep-dependent memory consolidation. Nature, 437, 1272-1278. doi: 10.1038/ nature 04286.

Stranges, S. et al. (2008). Correlates of short and long sleep duration: a cross-cultural comparison between the United Kingdom and the United States: the Whitehall II Study and the Western New York Health Study. American fournal of Epidemiology, 168, 1353-1364. doi: 10.1093/aje/ kwn337

Takano, K. and Tanno, Y. (2011) Diurnal variation in rumination. Emotion, 11, 1046e58. doi: 10.1037/a0022757

Van den Kommer, T. N., Comijs, H. C., Aartsen, M. J., Huisman, M., Deeg, D. J. and Beekman, A. T. (2013). Depression and cognition: how do they interrelate in old age? The American fournal of Geriatric Psychiatry, 21, 398-410

Van der Krieke, L. et al. (2017). Temporal dynamics of health and well-being: a crowdsourcing approach to momentary assessments and automated generation of personalized feedback. Psychosomatic Medicine, 79, 213-223. doi: 10.1097/PSY.0000000000000378 
Van der Krieke, L. et al. (2015). Ecological Momentary Assessments and Automated Time Series Analysis to Promote Tailored Health Care: A Proof-of-Principle Study. FMIR Research Protocols, 4, e100. doi: 10.2196/ resprot. 4000

Van Mill, J. G., Vogelzangs, N., van Someren, E. J., Hoogendijk, W. J. and Penninx, B. W. (2014). Sleep duration, but not insomnia, predicts the 2-year course of depressive and anxiety disorders. The fournal of Clinical Psychiatry, 75, 119-126. doi: 10.4088/JCP.12m08047.

Van Roekel, E., Vrijen, C., Heininga, V. E., Masselink, M., Bos, E. H. and Oldehinkel, A. J. (2017). An exploratory randomized controlled trial of personalized lifestyle advice and tandem skydives as means to reduce anhedonia. Behavior Therapy, 48, 76-96. doi: 10.1016/j .beth.2016.09.009

Xiang, X. and An, R. (2015). The impact of cognitive impairment and comorbid depression on disability, health care utilization, and costs. Psychiatric Services, 66, 1245-1248. doi: 10.1176/appi.ps.201400511.

Yoo, S., Gujar, N., Hu, P., Jolesz, F. A. and Walker, M. P. (2007). The human emotional brain without sleep - a prefrontal amygdala disconnect. Current Biology, 17, $1 \mathrm{e} 8$. doi: 10.1016/j.cub.2007.08.007

Zhou, F. et al. (2019). Regional cerebral hypoperfusion after acute sleep deprivation: a STROBE-compliant study of arterial spin labeling fMRI. Medicine (Baltimore), 98, e14008. doi: 10.1097/MD.0000000000014008. 